\title{
Testing Binary Population Synthesis Models with Hot Subdwarfs
}

\author{
Richard A. Wade*, Ravi kumar Kopparapu ${ }^{\dagger}$ and Richard O’Shaughnessy ${ }^{\dagger}$ \\ ${ }^{*}$ Dept. of Astronomy \& Astrophysics, Pennsylvania State University, 525 Davey Lab, University \\ Park PA 18602 USA \\ ${ }^{\dagger}$ Institute for Gravitational Physics and Geometry, Pennsylvania State University, 334 Whitmore \\ Lab, University Park, PA 16802-6300, USA
}

\begin{abstract}
Models of binary star interactions have been successful in explaining the origin of field hot subdwarf (sdB) stars in short period systems, but longer-period systems that formed via Rochelobe overflow (RLOF) mass transfer from the present $\mathrm{sdB}$ to its companion have received less attention. We map sets of initial binaries into present-day binaries that include sdBs and mainsequence stars, distinguishing "observable" sdBs from "hidden" ones. We aim to find out whether (1) the existing catalogues of sdBs are sufficiently fair samples of all the kinds of sdB binaries that theory predicts; or instead whether (2) large predicted hidden populations mandate the construction of new catalogues, perhaps using wide-field imaging surveys such as 2MASS, SDSS, and Galex. We also report on a pilot study to identify hidden subdwarfs, using 2MASS and GALEX data.
\end{abstract}

Keywords: binaries: close - stars: horizontal-branch - subdwarfs - ultraviolet: stars

PACS: $95.85 . \mathrm{Mt}$; $97.80 . \mathrm{Fk}$

\section{FORMATION CHANNELS FOR HOT SUBDWARF BINARIES}

Hot subdwarf B (sdB) stars are considered to be He-core burning systems with masses usually near $0.5 \mathrm{M}_{\odot}$ and thin hydrogen envelopes, located at the blue end of the horizontal branch. Since they form a narrow sequence, they are also referred to as Extended Horizontal Branch (EHB) stars. Studies by Maxted et al. [1] indicated that nearly half of the apparently single sdB stars are in short period binaries with white dwarf (WD) or M dwarf companions. The possible formation channels include stable, conservative mass transfer through a Roche lobe overflow (RLOF) or a dynamical-timescale mass transfer (unstable) leading to a common envelope (CE) evolution. Maxted's binaries are products of CE evolution. The RLOF channel can generate luminous main sequence (MS) companions at long periods, with sdB masses and luminosities lower than typical. How important is the RLOF channel?

We used the Binary Stellar Evolution (BSE) code of Hurley et al. [2], mapping sets of initial binaries into present-day binaries that include sdBs, and distinguishing "observable" sdBs from "hidden" ones. The common envelope efficiency parameter, $\alpha_{C E}$ was set to 1.5 . One million initial binaries were chosen at random using an initial mass function for $m_{2}$, uniform distribution of mass ratio $m_{1} / m_{2}<1$, and $\log$-uniform distribution of initial separation. We define a star as an sdB in the BSE code if it has surface gravity in the range $5.0<\log g<6.6(\mathrm{cgs})$ and effective temperature in the range $20,000 \mathrm{~K}<T_{\text {eff }}<45,000 \mathrm{~K}$. Systems that form sdB binaries with MS companions have initial values of $m_{1}$ between 0 and $5 \mathrm{M}_{\odot}$ depending on $m_{2}$. Initial orbital periods for 


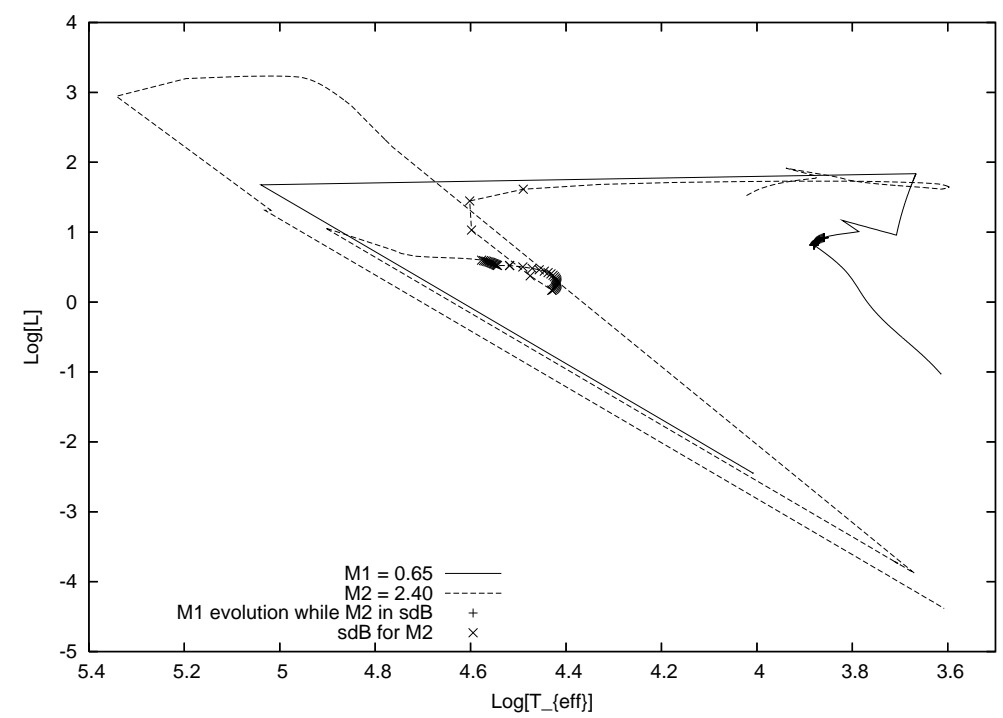

FIGURE 1. A $\left(\log T_{\text {eff }}, \log L\right)$ diagram illustrating the evolution of a binary system that forms an sdB star and an F0 companion.

these systems are between 1 and 200 days.

The distribution of $T_{\text {eff }}$ for the MS companions versus the orbital period after sdB formation shows two distinct sub-populations, reflecting the RLOF channel (long period, 2-300 d) and the CE channel (short period, 0.3-3 d). Similar work by Han et al. [3] shows an absence of systems with A-F type companions for orbital periods shorter than 10 days. Using the Hurley et al. [2] code, we do not find such a gap. Observations of a "fair sample" of sdB binaries should provide some insights.

\section{AN EXAMPLE BINARY WITH A HIDDEN HOT SUBDWARF}

Figure 1 shows the stellar evolutionary tracks for a binary system that forms an sdB star and a MS companion; initial masses were 0.65 and $2.40 \mathrm{M}_{\odot}$, the initial orbital period was 2.20 days. The more massive star (dashed line) evolves off the MS first and fills its Roche lobe to start a stable mass transfer onto its low mass MS companion, which becomes an F0 star. In the process, the first star loses its envelope to form a He-burning star, which we classify here as an sdB. Crosses/plusses mark the parts of the tracks of both stars while the initially more massive star is in the $\mathrm{sdB}$ phase. This stage of $\mathrm{sdB}+\mathrm{F} 0$ MS binary lasts for $\sim 1.0 \mathrm{Gyr}$, so such systems may be relatively numerous. The sdB stars exhausts $\mathrm{He}$ and becomes a CO WD. The F0 star remains on the MS for another $\sim 1.2$ Gyr. The system evolves further, after a CE episode, as a pair of low-mass WDs $(\mathrm{CO}+\mathrm{He})$ which coalesce at age $3.3 \mathrm{Gyr}$, briefly making a He star, then a CO WD.

Figure 2 presents brightness estimates at different wavelengths of the sdB star and its F0 companion from Fig. 1. The sdB star has lower than canonical mass $\left(0.319 \mathrm{M}_{\odot}\right)$, hence low luminosity and a long lifetime. The system has been placed at $1.0 \mathrm{kpc}$. Vertical bars indicate the approximate effective wavelengths and useful magnitude ranges of 




FIGURE 2. The spectral energy distributions of the sdB and F0 stars from Fig. 1. Vertical bars indicate the approximate effective wavelengths and useful magnitude ranges of GALEX AIS, SDSS, and 2MASS.

GALEX AIS, SDSS, and 2MASS. During the long sdB+F0 phase, the companion dominates the sdB in the optical, but not in the far UV.

Such systems typically would not be found in present catalogues of sdB stars. In the SDSS u'g'r'i'z' bandpasses, the companion star would be saturated at $1.0 \mathrm{kpc}$. At d > $1.0 \mathrm{kpc}$, where SDSS is not saturated, either one must observe near the Galactic plane (where SDSS and GALEX do not go and where reddening complicates the analysis) or one must observe several scale heights above the disk. A more effective way to search for such systems is to select F-star candidates based on their near-IR (2MASS) colors, then search for a far-UV excess (UVX) using GALEX. A preliminary search for F+UVX systems near $V \sim 12$ suggests that 1 or $2 \mathrm{~F}$ stars out of 100 may be hiding a hot subdwarf companion. We are observing a few of these at the Hobby-Eberly Telescope, to see whether the F star's radial velocity is variable. The ultimate goal is to measure the ratio of sdB binaries formed by the RLOF and CE channels in a fair sample, and to find the critical mass ratio that separates stable from unstable mass transfer.

\section{ACKNOWLEDGMENTS}

Supported by NASA NNG05GE11G and NSF PHY 03-26281, PHY 06-00953 and PHY 06-53462. This work was also supported by the Center for Gravitational Wave Physics, which is supported by the National Science Foundation under cooperative agreement PHY 01-14375.

\section{REFERENCES}

1. P. F. L. Maxted, U. Heber, T. R. Marsh., and R. C. North, M.N.R.A.S., 326, 1391-1402 (2001).

2. J. R. Hurley, C. A. Tout, and O. R. Pols, M.N.R.A.S., 329, 897-928 (2002).

3. Z. Han, Ph. Podsiadlowski, P. F. L. Maxted, and T. R. Marsh, M.N.R.A.S., 341, 669-691 (2003). 